\title{
Trimpi occurrence and geomagnetic activity: Analysis of events detected at Comandante Ferraz Brazilian Antarctic Station $(L=2.25)$
}

\author{
J. H. Fernandez, ${ }^{1,2}$ L. Rizzo Piazza, ${ }^{1,2}$ and P. Kaufmann ${ }^{1,3}$ \\ Received 13 December 2001; revised 29 July 2002; accepted 27 September 2002; published 28 January 2003.
}

[1] We present an analysis of the occurrence of Trimpi events observed at Comandante Ferraz Brazilian Antarctic Station (EACF), at $\mathrm{L}=2.25$, as observed by the amplitude of very low frequency (VLF) signals transmitted from Hawaii (NPM $21.4 \mathrm{kHz}$ ) from April 1996 to August 1999. The event parameters (total duration, amplitude variation, time incidence, and type (negative or positive)) were analyzed for 4394 events detected in the first year (solar minimum and relatively low Trimpi activity). The Trimpi occurrence was compared with geomagnetic activity for the whole period. A higher incidence of Trimpi events was observed near the equinoxes, with minima at the solstices following the same behavior as the Ap indices annual variation. During the day, their incidence peaked at $\sim 0700$ and $\sim 1045$ UT in accordance with the occurrence of lightning flashes in the geomagnetic conjugate regions but apparently not in agreement to the daily mean geomagnetic variation ( $\mathrm{Kp}$ index) found to be peaked (in entire years sampling average) at 0300 UT and weaker at 0900 UT for the whole 4-year period. The relationship between Trimpi occurrence and rise in solar activity for cycle 23 is discussed. A sudden strong incidence of Trimpi events around 5 May 1998 and 27 August 1998 was attributed to pronounced magnetic storms (Dst index). INDEX TERMS: 2487 Ionosphere: Wave propagation (6934); 2455 Ionosphere: Particle precipitation; 1640 Global Change: Remote sensing; 2435 Ionosphere: Ionospheric disturbances; KEYWORDS: ionosphere, Antarctic Peninsula, Trimpi events, geomagnetic activity, radio propagation

Citation: Fernandez, J. H., L. Rizzo Piazza, and P. Kaufmann, Trimpi occurrence and geomagnetic activity: Analysis of events detected at Comandante Ferraz Brazilian Antarctic Station $(\mathrm{L}=2.25)$, J. Geophys. Res., 108(A1), 1041, doi:10.1029/2001JA009213, 2003.

\section{Introduction}

[2] The Trimpi event is defined as an ephemeral amplitude and/or phase perturbation on very low frequency (VLF) radio signals propagating in the Earth-ionosphere waveguide due to an enhanced ionization patch produced by a burst of energetic electrons $(>40 \mathrm{keV})$, usually triggered by lightning. This is due to precipitation from the Van Allen radiation belts into the lower ionosphere [Helliwell et al., 1973]. These events can also be observed at low (LF) and medium (MF) radio frequencies. The classic manifestation of this phenomenon, caused by Lightning-Induced Electron Precipitation (LEP), is a consequence of the cyclotron resonance between whistler mode waves (generated from the lightning discharge) and counter-traveling electron fluxes from the radiation belts, in the Earth's magnetosphere, in a region near the geomagnetic equator. The

\footnotetext{
${ }^{1}$ Centro de Rádio Astronomiae Astrofísica Mackenzie (CRAAM), Instituto Nacional de Pesquisas Espaciais (INPE) Mackenzie, Universidade Presbiteriana Mackenzie, São Paulo, São Paulo, Brazil.

${ }^{2}$ Instituto Nacional de Pesquisas Espaciais (INPE), São José dos Campos, São Paulo, Brazil.

${ }^{3}$ Part time researcher at Centro de Componentes Semicondufores/ Universidade Estradual de Campinas (CCS/UNICAMP), Campinas, São Paulo, Brazil.
}

Copyright 2003 by the American Geophysical Union. 0148-0227/03/2001JA009213 interaction scatters energetic electrons to smaller pitch angles, putting them into the loss cone region and producing the precipitation burst [Inan et al., 1982]. The events generated by this mechanism are usually termed "classic" Trimpi events, while those generated by direct coupling of thundercloud fields with the ionosphere are designated as early/fast events due to their distinct characteristics, a premature (early) and rapid (fast) onset. Recently, Johnson et al. [1999a] have confirmed another class of events, the so-called "oblique" Trimpi events produced by obliquely propagating whistler waves that can propagate over a wide range of L-shells, and so interact with a larger population of trapped electrons in the radiation belts. They precipitate into the lower ionosphere to cause a bigger disturbed area.

[3] The classic Trimpi event at midlatitudes $(2<\mathrm{L}<3)$ consists of a rapid $(\sim 1 \mathrm{~s})$ change in amplitude and/or phase of the VLF radio signal received from a distant transmitter, followed by a relatively slower $(\sim 10-600 \mathrm{~s})$ recovery time describing an exponential curve profile [Inan and Carpenter, 1987]. In amplitude, the events can be positive (the onset corresponds to a sudden increase of the signal) or negative (a sudden decrease) (Figure 1), depending on the final result of the scattering on the azimuth of the signal arrival direction. The physical mechanisms that produce these features depend on several factors discussed in the next sections.

[4] In this work we are concerned with VLF amplitude Trimpi events detected at Comandante Ferraz Brazilian 

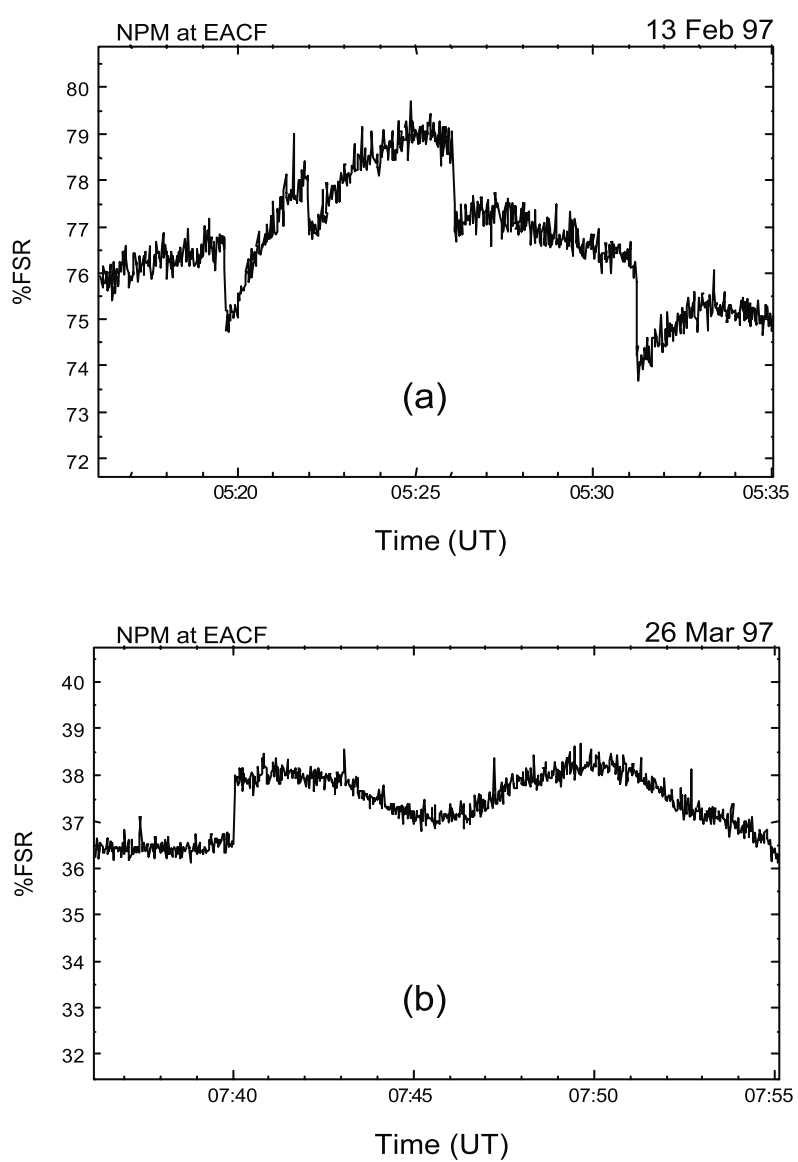

Figure 1. NPM amplitude signals recorded at EACF: (a) four negative Trimpi events on 13 February 1997 and (b) a positive Trimpi event on 26 March 1997.

Antarctic Station (EACF) located on King George Island, Antarctic Peninsula $\left(62^{\circ} 34^{\prime} \mathrm{S}, 58^{\circ} 23.5^{\prime} \mathrm{W} ; \mathrm{L}=2.25\right)$ in the period from April 1996 to August 1999. At this site the best VLF signal/noise ratio was obtained for NPM transmissions at $21.4 \mathrm{kHz}$ from Lualualei, Hawaii, USA $\left(21^{\circ} 25^{\prime} \mathrm{N}\right.$, $\left.158^{\circ} 09^{\prime} \mathrm{W}\right)$. The analyzed data were continuously recorded between 0000 and 1200 UT.

[5] The Antarctic Peninsula is an interesting location to detect classic and/or oblique Trimpi events since it is located at the East side of the South Atlantic Magnetic Anomaly, where electron loss occurs naturally as they drift in the westward direction. Another relevant factor is that the Antarctic Peninsula region is geomagnetically conjugate to very active North hemisphere thunderstorm centers [Rycroft, 1991].

\section{Data Analysis}

[6] We used a tunable VLF (TVLF) reception system, designed by Stanford University, with $10 \mathrm{~ms}$ resolution, similar to the instrumentation used by Johnson et al. [1999a, 1999b].

[7] The daily summary charts printed out were first analyzed with preenlargements of the probable events, and reduced using the MacTrimpi 5.2.1 software for MacOS developed by the Stanford University VLF Group. All the events with amplitude variation $0.25 \%$ Full-Scale Range (FSR) were selected as being above the threshold defining an event. In order to identify the special and statistical properties of the Trimpi event profiles at EACF and to derive their relevant parameters, namely, duration, time incidence, amplitude perturbation (in \%FSR) and sense (positive or negative), we selected 4394 events obtained in the period April 1996 to March 1997.

[8] To examine the statistical properties, only the first year of data was totally reduced to trace the Trimpi event profile at EACF because, in principle, it could be done with a complete period of seasonal variation. However, to study the relationship between Trimpi occurrence and geomagnetic activity, the whole of the 4-year period was used although without the extraction of all the event parameters.

\subsection{Trimpi Event Occurrence and Variation With Geomagnetic Activity}

[9] For the distribution of events detected with the time of the day, we have plotted their incidence as a function of UT time detection (LT $=\mathrm{UT}-4$ hours) with range intervals of 15 min (Figure 2). There is a concentration of events in two time intervals. The first one (more pronounced) is centered on $0700 \mathrm{UT}$ and the second on $\sim 1045 \mathrm{UT}$. The maxima can be best fitted by a double Gaussian plot resulting in $\sim 3$ hours width at half height for the first peak and $\sim 1.6$ hour for the second one. It can be also observed in Figure 2 that all the Trimpi events are concentrated in the period 04001200 UT, fitting these superimposed Gaussian distributions, similarly to the data obtained at Palmer Station, located near EACF [Carpenter and Inan, 1987]. The main reason for the low incidence before 0400 UT is that, for most of the year, a

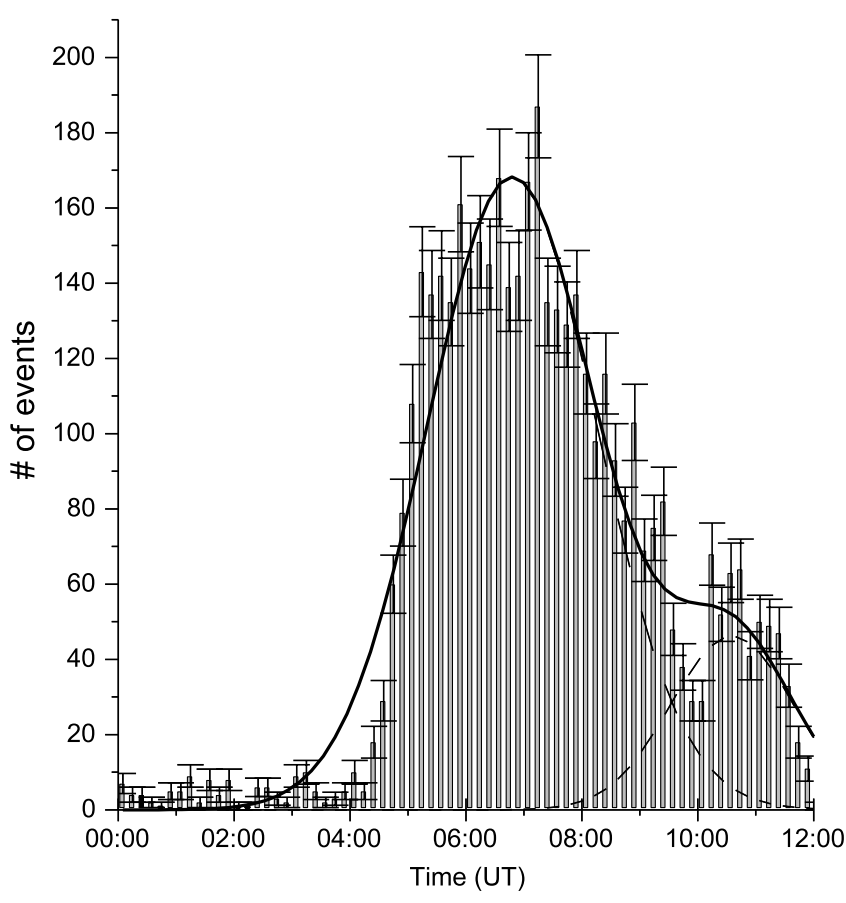

Figure 2. Histogram showing two regions of more probable occurrence fitted by a double Gaussian curve (the interval over which events are counted is $15 \mathrm{~min}$ ). 


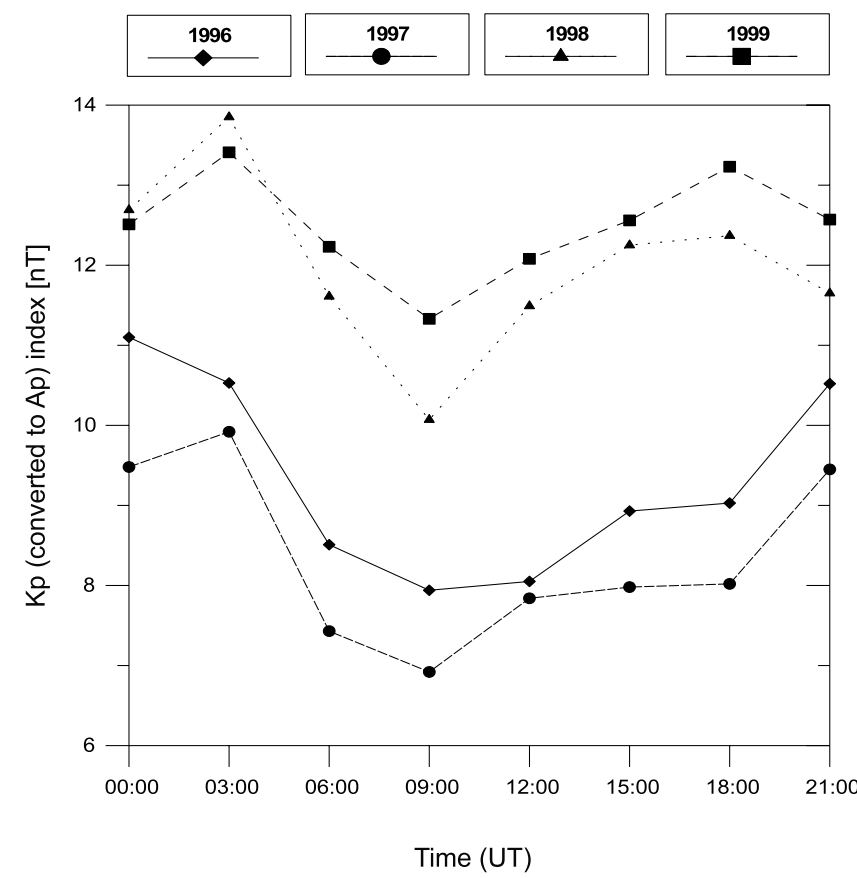

Figure 3. Kp (converted to Ap) index daily variation. Each time value is a result from the entire year sampling average.

large fraction of the NPM-EACF great circle path is under daytime conditions, inhibiting the detection of Trimpi event signatures because of the excess ionization due to the Sun which dominates the VLF amplitude level. The distribution found between 0700 and 1200 UT for the Trimpi activity is in accordance with the average distribution for the lightning flashes in the geomagnetic conjugate regions [Orville and Huffines, 2000; Zajac and Rutledge, 2000] (high whistler occurrence) but not in agreement with the daily mean geomagnetic variations ( 3 hour $\mathrm{Kp}$ index) peaked at 0300 UT and weaker at about 0900 UT (Figure 3). This daily curve was constructed through the entire year 3-hourly indices average, so each time value is a result from 365 (or 366 for 1996) days measurement at that specific time. The peak at about 0000-0300 UT corresponds to the Pacific Ocean predominant hemisphere being illuminated by the Sun and the minimum at 0900 UT corresponds to the hemisphere with the larger portion of emerged lands (Africa and Asia) being heated by the solar radiation. There are some correlations with this plot and the Carnegie curve [e.g., Rycroft et al., 2000] for the Earth's electric field diurnal variation. For example, the peak in the geomagnetic daily curve matches with the minimum in the Carnegie curve and the maximum in the Carnegie is associated with a local maximum in the geomagnetic daily variation. Finally, the minimum in the geomagnetic daily curve corresponds to an intermediate step (plateau) in the Carnegie curve. The time difference between the daily maximum Trimpi incidence and the daily geomagnetic variation ( $\sim 4$ hours) is compatible to the drift period for electrons with $80-100$ $\mathrm{keV}$ at $\mathrm{L}=2.25$.

[10] The Trimpi events detected from 1996 to 1999 were plotted, together with the geomagnetic activity, as shown

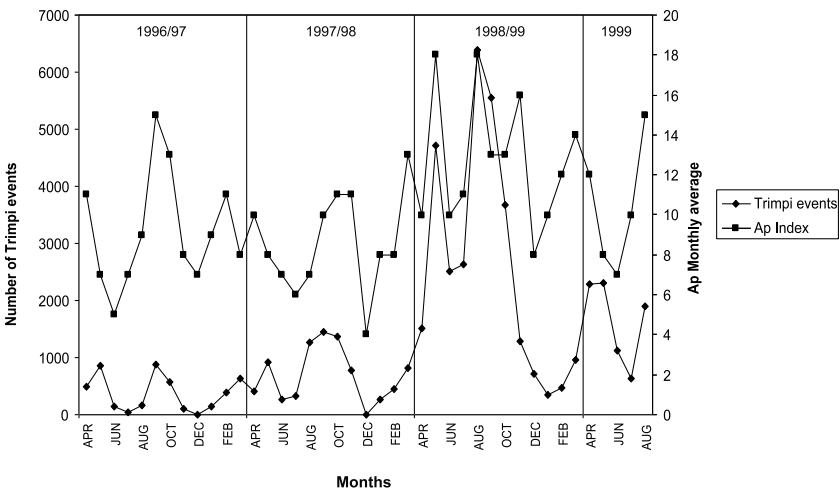

Figure 4. Comparison between monthly occurrence of Trimpi events and geomagnetic activity (in terms of Ap monthly average). The large increase, evident in August 1998 , is attributed to a strong magnetic storm coincident with a cosmic gamma-ray event.

in Figure 4. Both show similar variations with time. The two more prominent peaks in the Trimpi occurrence (in May 1998 and August 1998) are correlated with larger geomagnetic storms (Dst index) and the annual variation of the Dst index (Figure 5) shows this association; when the Dst (i.e., the magnitude of the geomagnetic field) is a minimum period the Trimpi occurrence is a maximum. We can interpret this fact remembering that a typical geomagnetic storm is characterized by a sudden increase in the magnitude of the geomagnetic field (due to the initial field line compression caused by the shock wave, this stage of the storm being called sudden commencement) followed by a stronger decrease (main phase). While the sudden commencement lasts a few hours, the main phase can last for a few days. During the main phase, the magnitude of the geomagnetic field is anomalously decreased due to ring

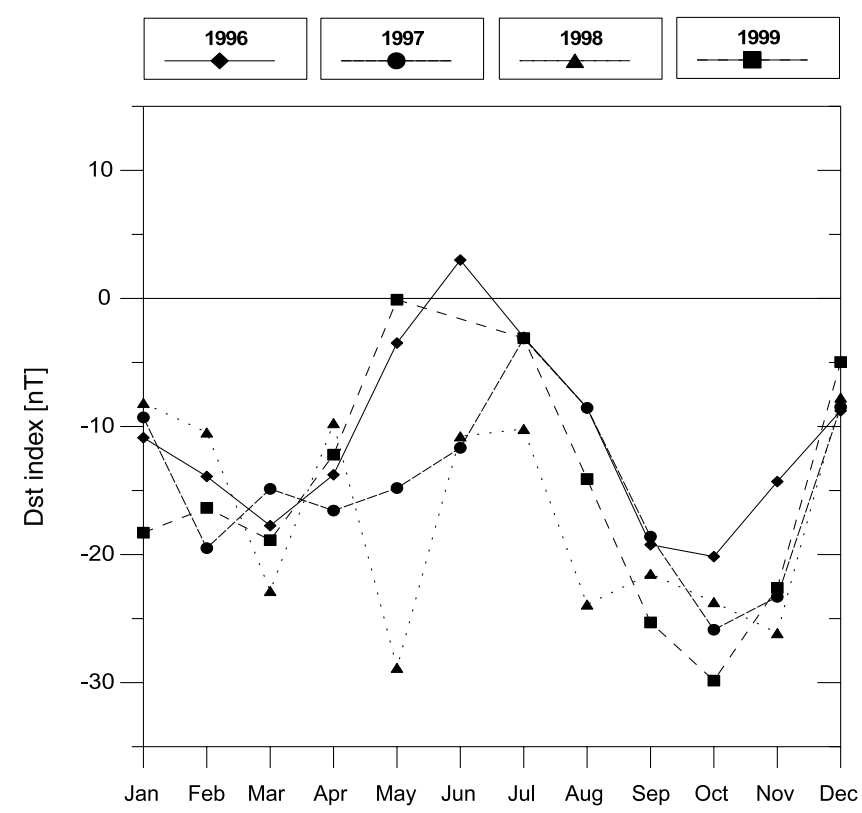

Figure 5. 3-hour $\mathrm{Kp}$ index (converted to $\mathrm{Ap}$ ) annual variation. The average was made during the entire year (from 1996 to 1999) for each time. 


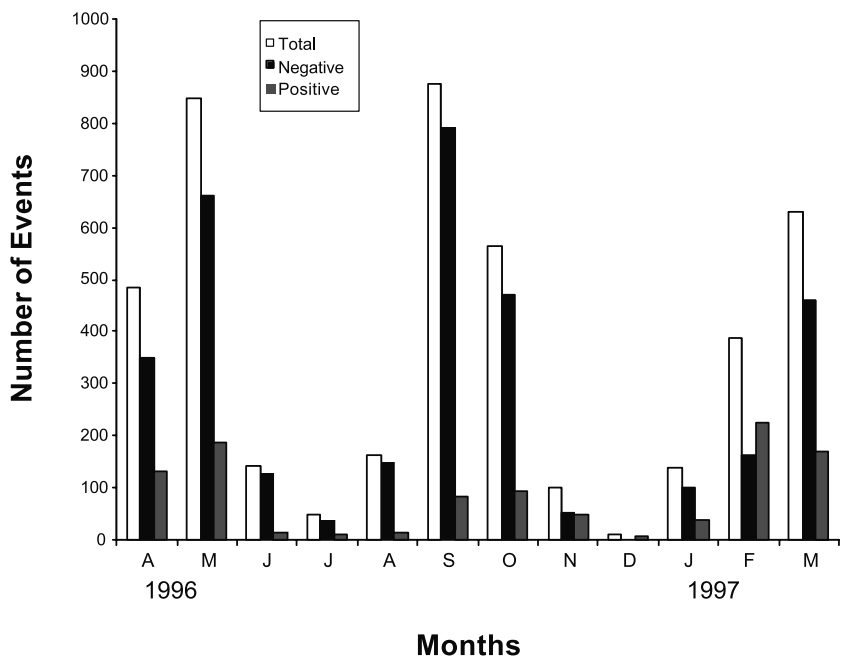

Figure 6. Seasonal variation of total (positive and negative) Trimpi events detected at Comandante Ferraz Station from 1 April 1996 to 31 March 1997. The equinoxial maxima are very clear.

currents that appear around the geomagnetic equator. These directly affect the mirror altitude of trapped electrons in the Van Allen radiation belts, facilitating the Trimpi event occurrence (the loss cone region is enlarged). Also, geomagnetic storms inject fluxes of particles (protons and electrons, accelerated from the solar wind) suddenly into the Earth's magnetosphere. This increases the population of particles that can be precipitated to cause a Trimpi event.

[11] For the first year (April 1996 to March 1997), the histograms for the total number (negative and positive events) per month detected during the period are shown in Figure 6 . We can identify a cyclic variation during the year with two maximum incidence periods, around the spring and autumn equinoxes, with minima at the solstices. In Figure 7 we show the percentage of Trimpi incidence for the

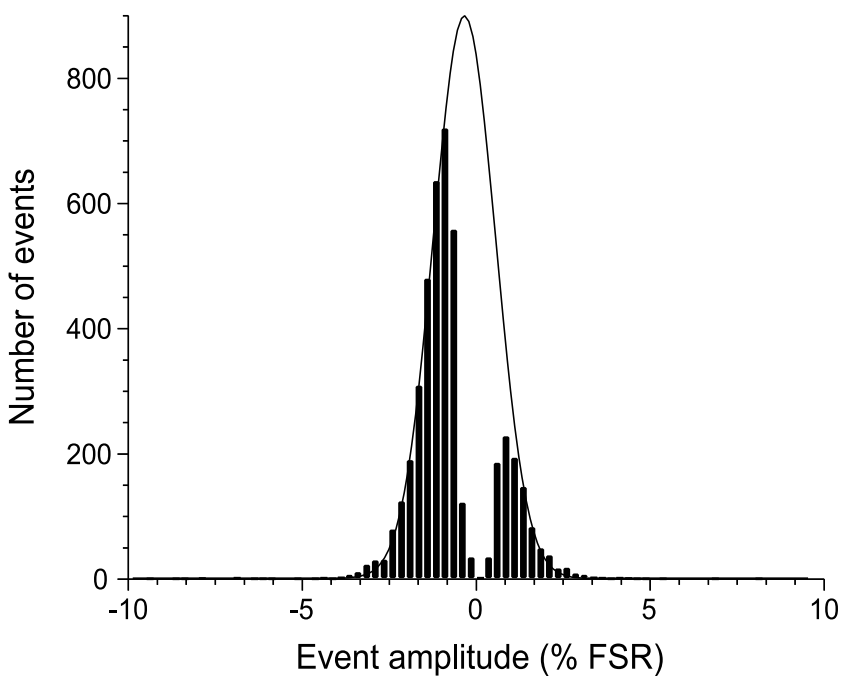

Figure 8. Distribution of Trimpi event amplitudes in $\%$ FSR (amplitude increments of $0.25 \%$ ) fitted by a single Gaussian curve.

months of the year when they were sampled. There is a clear excess of positive events for the local summer months.

\subsection{Distributions of Amplitude and Duration}

[12] The distribution for the incidence of Trimpi events with different amplitudes is shown in Figure 8. The absence of data for amplitudes close to zero is a consequence of the selection criteria. However, a single Gaussian fits the positive and negative amplitude samples well, with a maximum at about $\sim-0.5 \% \mathrm{FSR}$.

[13] Figure 9 shows the distribution of the total time duration of the Trimpi events. The duration corresponds to the entire time of the perturbation on the VLF signal, including the onset $(\sim 1-3 \mathrm{~s})$ and the recovery time (ionospheric recombination).

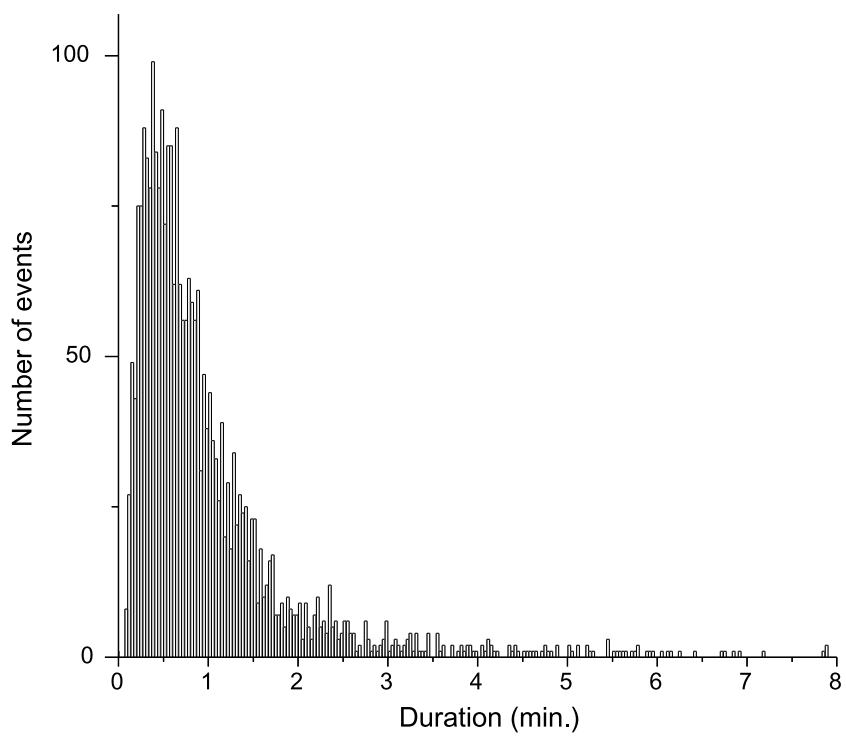

Figure 9. Histogram of Trimpi event durations. The

counting interval is $5 \mathrm{~s}$.
Figure 7. Relative occurrence of positive/negative events for the period April 1996 to March 1997.

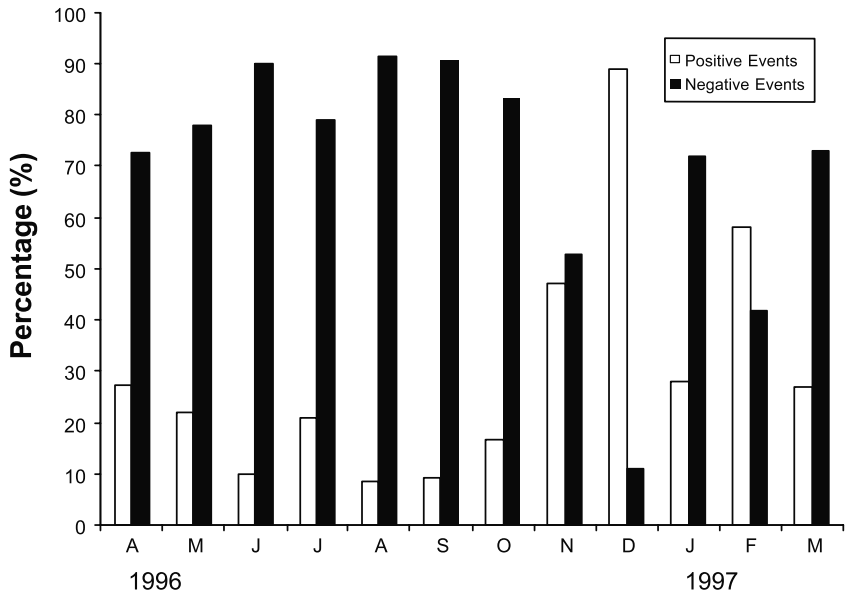

Months 


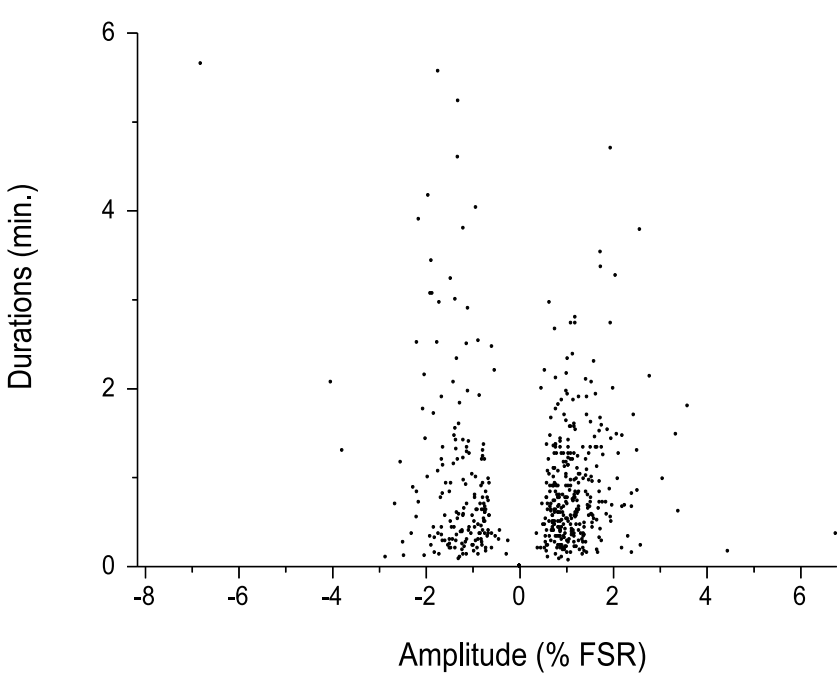

Figure 10. Trimpi event amplitude versus duration scatter diagram.

[14] The distribution suggests a real maximum for durations around $30 \mathrm{~s}$. The reduction in probability for durations $<30$ s cannot be attributed to the event selection criteria. There is no good correlation between duration and amplitude, as shown in the scatter diagram of Figure 10.

\subsection{Solar Cycle Effects and the 1998 Cosmic Gamma Ray Event}

[15] For the period April 1996 to March 1997 the total number of the Trimpi perturbations observed was 4394. In the following year, from April 1997 to March 1998, the number increased to 8333 and, for April 1998 to March 1999 was 30,734 (see Figure 11), showing a significant rise. The number of Trimpi events accumulated per year correlates well with the total number of solar flares (upper right corner in the Figure 11).

[16] The year 1998 showed a Trimpi activity curve profile not directly related to solar activity, but well correlated with geomagnetic activity (Figure 4). The periods with high Trimpi incidence, in May and August 1998, were related to strong geomagnetic storms.

[17] An intense cosmic gamma-ray burst was observed in the direction of the Aquila constellation on 27 August 1998 [Inan et al., 1999]. However, its effects on the August enhancements in Trimpi events occurrence cannot be evaluated because the whole period was dominated by the strong geomagnetic storm.

\section{Discussion}

[18] The maximum occurrence of Trimpi events near the equinoxes, shown in Figure 6, might be explained as being due to the higher "stability" of the magnetic line configuration. In these seasons, the solar wind and solar radiation reach both hemispheres most uniformly (at smaller angles with respect to the geomagnetic equator). Thus, the equatorial gyroresonance mechanism is more symmetric. Another reason might be attributed to the $7.2^{\circ}$ tilt of the solar rotation axis with respect to the normal to the ecliptic; the Earth reaches the highest northern and southern helio- graphic latitudes (where the solar wind speed is higher) on 6 September and 5 March, respectively, and crosses the equator twice a year between these dates. Thus, when observed from the Earth, we expect a semiannual variation in the solar wind velocity and electrically charged particles trapped in the Van Allen radiation belt, the "source" of the classic and oblique Trimpi phenomena, with maxima around these dates. The annual variation is often clearer [e.g., Bolton, 1990], because the solar wind distribution is asymmetric or shifted with respect to the equator [Zieger and Mursula, 1998].

[19] The minimum in the austral summer is expected because the ionosphere over Antarctica is continuously illuminated by the Sun; the reduced lightning source activity in the geomagnetically conjugate region also contributes. As the thunderstorm occurrence is higher in the local summer season, there is a reduction in whistler production in the boreal winter and so it is reasonable to expect a decrease in the Trimpi occurrence in the correspondent austral summer. Similar reasons explain the occurrence of Trimpi events being higher in the austral winter than in the austral summer (the daytime period being shorter and the lightning occurrence a maximum in the geomagnetically conjugate region). On the other hand, the occurrence rate in the austral winter is not high enough to be stronger than the equinox incidence due to the solstice "instability" (in terms of the geometry of the gyroresonance mechanisms).

[20] The larger probability for negative amplitude events, shown in Figure 7, might be because the NPM-EACF great circle propagation path is located completely over the Pacific Ocean as pointed out by Inan and Carpenter [1987]. The propagation of VLF wave modes in the Earth-ionosphere waveguide has been considered in detail by Budden [1961] and Wait [1962]. At VLF, only a few modes are important; the higher-order modes are attenuated

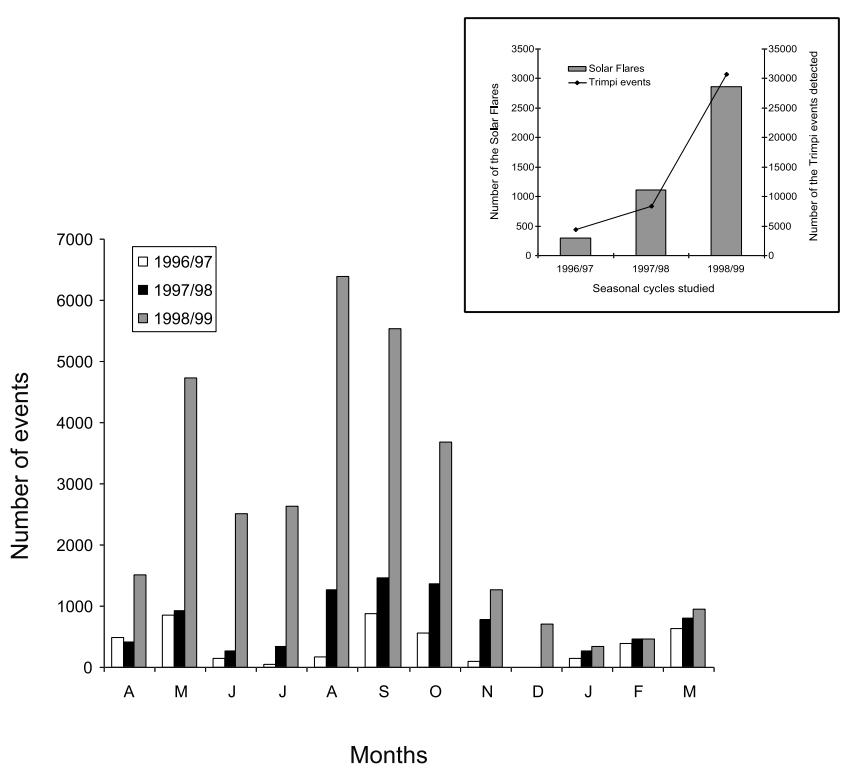

Figure 11. Comparison between Trimpi activity through the year for the 3 years. In the upper right corner, the total number of Trimpi events detected each year is compared with the incidence of solar flares. 
at a much greater rate than the lower-order modes, so that at great distances only the first mode (mode 1) need to be considered. The predominance of the mode 1 in the Earthionosphere waveguide might explain the higher incidence for negative events observed, also as proposed by Inan and Carpenter [1987] who analyzed data obtained at Palmer Station near EACF. Another possible explanation given by Friedel and Hughes [1990] who studied data recorded at Sanae Station $\left(71^{\circ} 41^{\prime} \mathrm{S}, 02^{\circ} 50^{\prime} \mathrm{W}\right)$ was that negative events were more common during disturbed periods. However, for the data analyzed in the first year studied (April 1996 to March 1997), magnetic activity was low, on average, as can be seen from Figure 6 . We can conclude that during disturbed periods the overall Trimpi activity increases and, with the negative type being more common (or in other words, more probable) for the NPM-EACF events, the relative percentage also changes in benefit of negative events.

[21] Poulsen et al. [1990] proposed that the negative or positive sense of event amplitude is mainly related to the transverse distance of the disturbed region from the VLF propagation path, with positive events due to the disturbed region being further from the great circle. Lev-Tov et al. [1995] suggested that the negative/positive behavior depends on several other factors such as the whistler intensity, trapped and precipitated energetic electron spectra/fluxes, the spatial extent of the event (determined by the size of the whistler mode propagation duct footprint and, as recently discovered [Johnson et al., 1999a; Lauben et al., 1999], by the angle of the whistler wave vector) and the ambient ionospheric electron density (which determines the VLF reflection height). So, a specific set of all these factors will actually determine the event's final signature and the sign of the VLF amplitude change.

[22] The electron energy was found to be around of 0.1-1 MeV, determined through the joint analysis from Comandante Ferraz and Palmer (USA) Stations of the same events (events originating in the same sferic, the same lightning source; work on this with Stanford University still has to be submitted for publication). The delay time between the event onset and the associated sferic has revealed that Comandante Ferraz events occur earlier, with a shorter delay ( $300 \mathrm{~ms})$ than Palmer ones (1-1.5 s). From these results and using the Lauben [1998] results we deduce that, for Palmer Station, the events originate from electrons precipitated at higher L shells while for Comandante Ferraz the precipitating electrons are from lower L-shells, where the gyroresonant electrons are more energetic, and the whistler wave propagation velocity is also higher.

[23] Finally, the real lower limit found for the duration of Trimpi events, around $30 \mathrm{~s}$, is the typical ionospheric recombination time at the heights $(80-100 \mathrm{~km}$, nighttime conditions) where the phenomena occur [Bilitza, 1997]. Even if an energetic burst (producing a larger amplitude disturbance) reaches lower altitudes, the dominant fraction of the recombination time is due to the chemical recombination processes at that heights (the lower portion of the disturbance besides be constituted of a smaller perturbation in ambient plasma density also has a faster chemical recombination). The strong scattering in the plot amplitude versus time duration seems to indicate that we have col- lected events with different horizontal sizes (classics and obloquies).

\section{Final Remarks}

[24] Most of the results on the seasonal variation of Trimpi event occurrence presented here are consistent with the result of Carpenter and Inan [1987] who found maximum occurrence around 0800 UT for the NPM signal received at Palmer Station $\left(65^{\circ} \mathrm{S}, 64^{\circ} \mathrm{W}, \mathrm{L} \sim 2.4\right)$ during 1983. They also observed the low incidence between 0000 and 0400 UT. The only disagreement was in the austral winter where they found a localized cluster of active days explained as being due to unusual magnetic activity.

[25] The range of amplitude perturbations was very similar to that obtained by Friedel and Hughes [1990], and exhibited an exponential decrease especially for larger amplitude disturbance events (in \%FSR) following the chemical recombination processes in the ionosphere at the heights where the events occur.

[26] Both geomagnetic activity and the occurrence of Trimpi events depend strongly on various space weather parameters, as, for example, the radiation (electromagnetic or particles) processes occurring in the geospace. Although the Sun is the main disturbing agent, other events (like the 27 August 1998 cosmic gamma ray event) might disturb the ionosphere as well.

[27] This research will continue at EACF to investigate the long-term properties of Trimpi ionospheric disturbances in order to better understand the ionosphere over the Antarctic Peninsula and to try to elucidate the mechanisms operating in the Van Allen radiation belts as well as their space weather variations.

[28] Acknowledgments. We would like to thank U. S. Inan, J. Yarbrough, and the VLF Group from Stanford University for their contribution and constant support and all researchers and technicians who operated the equipment at EACF. We acknowledge the useful comments and corrections in the text given by the two referees.

[29] This work was partially sponsored by the Brazilian Antarctic Program (PROANTAR), São Paulo State Research Support Foundation (FAPESP process 97/14578-0) and a Brazilian Federal Science and Research Support Agency (CAPES).

\section{References}

Bilitza, D., International reference ionosphere-Status 1995/96, Adv. Space Res., 20(9), 1751-1754, 1997.

Bolton, S., One year variation in the near Earth solar wind ion density and bulk flow velocity, Geophys. Res. Lett., 17, 37-40, 1990.

Budden, K. G., The Waveguide Mode Theory of Wave Propagation, Logos Press, London, UK, 1961.

Carpenter, D. L., and U. S. Inan, Seasonal, latitudinal and diurnal distributions of whistler-induced electron precipitation events, J. Geophys. Res., 92, 3429-3435, 1987.

Friedel, R. H. W., and A. R. W. Hughes, Characteristics and frequency of occurrence of Trimpi events recorded during 1982 at Sanae, Antarctica, J. Atmos. Terr. Phys., 52, 329-339, 1990.

Helliwell, R. A., J. P. Katsufrakis, and M. L. Trimpi, Whistler-induced amplitude perturbation in VLF propagation, J. Geophys. Res., 78, 4679-4688, 1973.

Inan, U. S., T. F. Bell, and H. C. Chang, Particle precipitation induced by short-duration VLF waves in the magnetosphere, J. Geophys. Res., 87, $6243-6264,1982$.

Inan, U. S., and D. L. Carpenter, Lightning-induced electron precipitation events observed at $\mathrm{L} \sim 2.4$ as phase and amplitude perturbations on subionospheric VLF signals, J. Geophys. Res., 92, 3293-3303, 1987.

Inan, U. S., N. G. Lehtinen, S. J. Lev-Tov, M. P. Johnson, T. F. Bell, and $\mathrm{K}$. Hurley, Ionization of the lower ionosphere by gamma-rays from a 
magnetar: Detection of a low energy $(3-10 \mathrm{keV})$ component, Geophys. Res. Lett., 26, 3357-3360, 1999.

Johnson, M. P., U. S. Inan, and D. S. Lauben, Subionospheric VLF signatures of oblique (nonducted) whistler-induced precipitation, Geophys. Res. Lett., 26, 3569-3572, 1999a.

Johnson, M. P., U. S. Inan, S. J. Lev-Tov, and T. F. Bell, Scattering pattern of lightning-induced ionospheric disturbances associated with early/fast VLF events, Geophys. Res. Lett., 26, 2363-2366, 1999b.

Lauben, D. S., Precipitation of radiation belt electrons by obliquely propagating lightning-generated whistler waves, Ph.D. thesis, Dept. of Electr. Eng., Stanford Univ., Stanford, Calif., October 1998.

Lauben, D. S., U. S. Inan, and T. F. Bell, Poleward-displaced electron precipitation from lightning-generated oblique whistlers, Geophys. Res. Lett., 26, 2633-2636, 1999.

Lev-Tov, S. J., U. S. Inan, and T. F. Bell, Altitude profiles of localized D region density disturbances produced in lightning-induced electron precipitation events, J. Geophys. Res., 100, 21,375-21,383, 1995.

Orville, R. E., and G. R. Huffines, Cloud-to-ground lightning in the United States: NLDN results in the first decade, 1989-98, Mon. Weather Rev. 129(5), 1179-1193, 2000.

Poulsen, W. L., T. F. Bell, and U. S. Inan, Three-dimensional modeling of subionospheric VLF propagation in the presence of localized D region perturbations associated with lightning, J. Geophys. Res., 95, $2355-$ 2366, 1990.

Rycroft, M. J., Interactions between whistler mode waves and energetic electrons in coupled system formed by the magnetosphere, ionosphere and atmosphere, J. Atmos. Terr. Phys., 53, 849-858, 1991.

Rycroft, M. J., S. Israelsson, and C. Price, The global atmospheric electric circuit, solar activity and climate change, J. Atmos. Sol. Terr. Phys., 62, $1563-1576,2000$.

Wait, J. R., Electromagnetic Waves in Stratified Media, Pergamon, New York, 1962.

Zajac, B. A., and S. A. Rutledge, Cloud-to-ground lightning activity in the contiguous United States from 1995 to 1999, Mon. Weather Rev., 129(5), 999-1019, 2000

Zieger, B., and K. Mursula, Annual variation in near-Earth solar wind speed: Evidence for persistent north-south asymmetry related to solar magnetic polarity, Geophys. Res. Lett., 25, 841-844, 1998.

J. H. Fernandez, P. Kaufmann, and L. Rizzo Piazza, CRAAM (Instituto Nacional de Pesquisas Espaciais (INPE) Mackenzie), Universidade Presbiteriana Mackenzie, R. da Consolação, 896, 01302-907, São Paulo, SP, Brazil. (jhenrix@aol.com) 